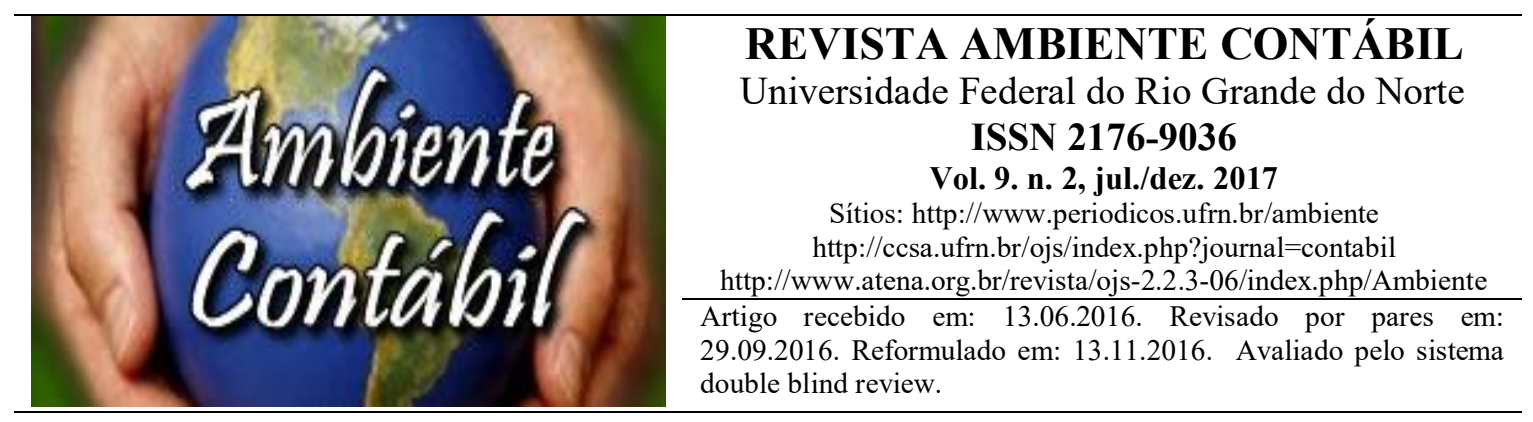

\title{
PRÁTICAS ORÇAMENTÁRIAS APLICADAS EM CONCESSIONÁRIAS DE VEÍCULOS NO ESTADO DE SANTA CATARINA
}

\author{
BUDGET PRACTICES APPLIED IN VEHICLE DEALERS IN SANTA CATARINA \\ STATE
}

\section{PRESUPUESTO EN PRÁCTICA APLICADO A LOS DISTRIBUIDORES DEL VEHÍCULOS EN EL ESTADO DE SANTA CATARINA}

Autores

Myrna Modolon Lima Mestranda do Programa de Pós-Graduação em Contabilidade (PPGC). Endereço: Rua Deputado Antônio Edu Vieira, 1422 - Apto 725 - Bairro Pantanal - Florianópolis-SC Brasil. Telefone: (48) 9985-2393- CEP: 88040-000.

Identificadores (ID): ORCID: http://orcid.org/0000-0003-2084-481X Lattes: http://lattes.cnpq.br/9859212802759845

E-mail:mmodolonlima@gmail.com

José Luiz Vailatti

Mestrando do Programa de Pós-Graduação em Contabilidade (PPGC). Professor do Instituto Fayal de Ensino Superior (IFES). Endereço: Rua Lico Amaral, 205 - Apto 65. Endereço: Bairro Dom Bosco - Itajaí-SC - Brasil. CEP 88307-010. Telefone: (47) 8402-5649. Identificadores (ID): ORCID: http://orcid.org/0000-0003-1744-054X Lattes: http://lattes.cnpq.br/6100612843784484 E-mail: vailatti@conjel.com.br

Rogério João Lunkes

Pós-Doutor em Contabilidade pela Universitat de Valencia, Espanha. Professor dos Programas de Pós-Graduação em Administração (PPGA) e Contabilidade (PPGC) da Universidade Federal de Santa Catarina (UFSC). Endereço: Campus Universitário Reitor David Ferreira Lima, Trindade - CEP: 88040-380 - Florianópolis - SC - Brasil. Telefone:

(48) 3721-6634. Identificadores (ID):

Redalyc: http://www.redalyc.org/autor.oa?id=11068 ORCID: http://www.orcid.org/0000-0003-4232-5746 Google Citations: https://scholar.google.com.br/citations?user=8-aGBSwAAAAJ\&hl=pt-BR Research Gate: https://www.researchgate.net/profile/Rogerio_Lunkes 
Myrna Modolon Lima, José Luiz Vailatti, Rogério João Lunkes, Valdirene Gasparetto e Darci Schnorrenberger

Lattes: http://lattes.cnpq.br/6241003358183170

E-mail: lunkes@cse.ufsc.br

Valdirene Gasparetto

Doutora em Engenharia de Produção pela Universidade Federal de Santa Catarina.

Professora adjunta da Universidade Federal de Santa Catarina.

Endereço: Departamento de Contabilidade - UFSC, Campus Universitário - Trindade, Florianópolis / SC - Brasil. Telefone: (48) 8404-2416. CEP: 88040-900.

Identificadores (ID):

Google Citations:https://scholar.google.com.br/citations?user=fhHVR5wAAAAJ\&hl=pt-BR

Research Gate: https://www.researchgate.net/profile/Valdirene_Gasparetto

Lattes: http://lattes.cnpq.br/9876735629466934

E-mail: valdirene.gasparetto@ufsc.br

\begin{abstract}
Darci Schnorrenberger
Doutor em Engenharia de Produção pela Universidade Federal de Santa Catarina. Professor do Programa de Pós-Graduação em Contabilidade da Universidade Federal de Santa Catarina. Endereço: Departamento de Contabilidade - UFSC, Campus Universitário - Trindade, Florianópolis / SC - Brasil. CEP: 88040-900 - Telefone: (48) 3721-6634. Identificadores (ID): Google Citations: https://scholar.google.com.br/citations?user=TOxIbYQAAAAJ\&hl=pt-BR Research Gate: https://www.researchgate.net/profile/Darci_Schnorrenberger Lattes: http://lattes.cnpq.br/1258009069271071 E-mail: darci.schnorrenberger@ufsc.br
\end{abstract}

\title{
RESUMO
}

O objetivo deste artigo é analisar as práticas de orçamentos em concessionárias de veículos em Santa Catarina. A pesquisa visa cobrir uma gama de questões de orçamento incluindo: o processo utilizado na definição do orçamento, acompanhamento e avaliação dos processos orçamentários e a execução e controle do orçamento. Para tanto foi realizada uma pesquisa descritiva, com abordagem quantitativa, com a utilização do questionário fechado de práticas de orçamento que foram respondidas por 20 (vinte) Concessionárias de Veículos em Santa Catarina. Conclui-se que a principal razão da elaboração do orçamento é para auxiliar no controle, avaliação de desempenho e no planejamento. A abordagem de elaboração conjunta é a mais utilizada pelas concessionárias. Os orçamentos são geralmente vistos como o principal indicador de desempenho das concessionárias. A aplicação prática do estudo é a recomendação para um processo orçamentário que melhore a precisão e melhore a eficiência de controle dos custos.

Palavras-Chaves: Práticas de Orçamento. Previsão. Concessionárias de Veículos.

\begin{abstract}
The purpose of this article is to analyze the practices of budgets at car dealerships in Santa Catarina. The research aims to cover a range of budget issues including: the process used for determining the budget, monitoring and evaluation of budgetary processes and control of the budget. For this purpose a descriptive survey was conducted with a quantitative approach, using the questionnaire enclosed budget practices that were answered by twenty (20) Vehicle Dealers in Santa Catarina. It is concluded that the main reason for budgeting is to help control, performance evaluation and planning. The joint development approach is the most
\end{abstract}


widely used by utilities. Budgets are usually seen as the main performance indicator for utilities. The practical application of the study is the recommendation for a budget process that improves the accuracy and improve cost control efficiency.

Keywords: Budget Practices, Forecasting, Vehicle Dealers

\section{RESUMEN}

El objetivo de este trabajo es analizar las prácticas de los presupuestos en los concesionarios de automóviles en Santa Catarina. La investigación tiene como objetivo cubrir una serie de cuestiones presupuestarias, incluyendo: el proceso utilizado para la determinación del presupuesto, seguimiento y evaluación de los procesos presupuestarios y la ejecución y control del presupuesto. Para dicho estudio descriptivo se llevó a cabo con un enfoque cuantitativo, utilizando las prácticas presupuestarias cuestionario cerrado que fueron contestadas por veinte (20) Vendedores de Vehículos en Santa Catarina. Se concluye que la razón principal para la elaboración del presupuesto es ayudar a control, evaluación del rendimiento y planificación. El enfoque de desarrollo conjunto es el más ampliamente utilizado por empresas de servicios públicos. Los presupuestos son generalmente vistos como el principal indicador de rendimiento de los servicios públicos. La aplicación práctica de este estudio es la recomendación para un proceso presupuestario que mejora la precisión y mejorar la eficiencia del control de costes.

Palabras clave: Prácticas presupuestarias. Pronóstico. Vendedores de Vehículos.

\section{INTRODUÇÃO}

A necessidade de planejamento e a crescente concorrência, compelem as empresas a buscarem práticas de gestão que agreguem resultados positivos. As organizações, com o intuito de dar eficácia a seus planejamentos, aderem a técnicas de gestão. Visto que, sejam empresas de pequeno ou grande porte, agências governamentais ou simplesmente para o controle pessoal, o planejamento torna-se imprescindível para a gestão (LEITE et al., 2008; LUNKES et al., 2014).

Dentre as práticas de gestão usualmente aplicadas nas empresas, destaca-se o orçamento como ferramenta gerencial. Com o intuito de auxiliar no gerenciamento da empresa, recomenda-se a utilização do orçamento de forma plena, difundindo-se seu significado por toda a organização (FRUTTICHER et al., 2005; PEREIRA et al., 2015).

Com êxitos e insucessos, o interesse pelo orçamento solidifica-se na literatura, em que se abordam o planejamento, execução e controle das práticas orçamentárias. Muitas vezes criticado por sua utilização inadequada nas organizações, ainda assim existem muitos entusiastas dessa prática gerencial, visto que tanto na literatura como nas empresas, pesquisase e defende-se sua utilização. (SCHMIDGALL E NINEMEIER, 1989; SCHMIDGALL et al., 1996; SCHMIDGALL E DEFRANCO, 1998; JONES, 1998; SUBRAMANIAM et al., 2002; WINATA E MIA, 2005; YUEN, 2006; JONES, 2008B; PAVLATOS E PAGGIOS, 2009; STEED E GU, 2009; UYAR E BILGIN, 2011; CASTRO et al., 2012; CODESSO, LUNKES, SUAVE (2013), LUNKES; CODESSO; RIPOLL-FELIU, 2014; LUNKES et al., 2014; PEREIRA et al., 2015).

Dentre as empresas que se utilizam de práticas de gestão, estão as concessionárias de veículos, que são organizações identificadas com as montadoras, realizando um tipo de distribuição exclusiva, que o fabricante elege para trabalhar só com seu mix de produtos (PAPA e RIQUENA, 2011). Além disto, é importante no negócio da distribuição de veículos a gestão profissional, uma vez que a operação exige profundo conhecimento técnico, aplicação de referências e parâmetros que apontem com segurança, tendências, resultados desvios, falhas e potenciais. Uma gestão profissional deve conseguir prever os próximos 
passos, buscar soluções para os problemas de forma rápida e eficiente, e inovar para poder competir em um mercado cada vez mais competitivo (PAPA; RIQUENA, 2011).

Pensando-se no planejamento das concessionárias de veículos e na funcionalidade dessa prática gerencial, surge a necessidade de conhecer a utilização e relevância do orçamento nestas empresas. Baseando-se na literatura preexistente referente às práticas orçamentárias, conduziu-se o estudo que respondesse ao seguinte questionamento: quais as práticas de orçamento utilizadas nas concessionárias de veículos em Santa Catarina? $O$ estudo apresenta como objetivo geral identificar e analisar as práticas de orçamento em concessionárias de veículos em Santa Catarina e comparar com as principais práticas de orçamento encontradas na literatura.

Justifica-se a pesquisa devido à relevância do setor de concessionária de veículos para o país, onde em 2014 representava 5,2\% do Produto Interno Bruto. Além disso procura-se aplicar o conhecimento existente sobre práticas de orçamentos, abrangendo também o setor de distribuição de veículos, já que há poucos estudos relacionados ao orçamento para esse seguimento da economia.

Além desse tópico introdutório, o estudo divide-se em mais quatro seções. A primeira apresenta o referencial teórico alusivo ao tema de orçamento e às práticas orçamentárias. $\mathrm{O}$ tópico seguinte aborda a metodologia utilizada no artigo. Em seguida, evidenciam-se e discutem-se os resultados encontrados. Por fim, apontam-se as principais conclusões relativas ao estudo e às referências bibliográficas.

\section{REFERENCIAL TEÓRICO}

Diversos autores (CASTRO et al., 2012; CODESSO; LUNKES; SUAVE, 2013, LUNKES; CODESSO; RIPOLL-FELIU, 2014; LUNKES et al., 2014; PEREIRA et al., 2015) abordam o orçamento e as práticas orçamentárias com foco na gestão das empresas (LEITE et al., 2008). Alguns defendem que o orçamento deve ser dividido em planejamento e controle (WELSCH, 1983; STEED; GU, 2007), enquanto outros argumentam que o orçamento deve ser dividido em planejamento, execução e controle (LUNKES, 2007; FREZATTI, 2009). Por acreditar que a divisão em três estágios proporciona uma melhor visualização e compreensão do funcionamento do orçamento nas organizações, opta-se por essa abordagem no presente estudo.

Nesta seção são apresentados, previamente, aspectos sobre as práticas de orçamento desde o planejamento, a execução e por fim o controle.

\subsection{PRÁTICAS DE ORÇAMENTO}

A literatura apresenta diversas pesquisas sobre orçamento e práticas orçamentárias. As práticas aplicam-se no planejamento, execução e controle orçamentários. Para isso, classificam-se quais as ferramentas utilizadas em cada etapa. Tal como a elaboração de manual e comissão de orçamento durante o planejamento, a avaliação do processo orçamentário na execução e utilização de benchmarking para a etapa de controle. Schmidgall e Ninemeier (1989) depararam-se com 65\% e 75\% de utilização dessa prática em hotéis e cadeias de serviços de alimentação nos Estados Unidos da América, respectivamente. Pavlatos e Paggios (2009), baseados nessa pesquisa, investigaram as práticas orçamentárias na indústria hoteleira grega, obtendo resultados satisfatórios referentes às adoções das práticas. Steed e Gu (2009) também corroboram dessa tendência na indústria hoteleira, com adoção quase plena nas redes hoteleiras entrevistadas.

Além do ramo hoteleiro, Joshi, Al-Mudhaki e Bremser (2003), identificaram práticas orçamentárias em empresas de Bahrain, principalmente referentes ao planejamento. No Brasil, 
pesquisas sobre práticas orçamentárias foram realizadas para o ramo hoteleiro (CODESSO; LUNKES; SUAVE, 2013; CASTRO et al., 2012), como também para empresas cerâmicas (DAITX; SCHNORRENBERGER, 2014), cooperativas agropecuárias (PEREIRA et al., 2015), empresas do setor de bens industriais (LUNKES et al., 2014), bancos comerciais públicos (SUAVE et al., 2013) e supermercados (CODESSO et al., 2015).

$\mathrm{Na}$ sequência, será apresentada a revisão da literatura que trata o planejamento e elaboração do orçamento, a execução e o controle.

\subsection{PLANEJAMENTO E ELABORAÇÃO DO ORÇAMENTO}

Compreendem-se as práticas inicias durante o planejamento e elaboração do orçamento. O manual de orçamento figura como uma das principais práticas, visto que apresenta os objetivos, metas e diretrizes estabelecidos pela organização (LUNKES et al., 2014). O Quadro 1, elaborado em concordância com os estudos de Codesso, Lunkes, Suave (2013), apresenta as principais práticas de planejamento e elaboração de orçamento encontradas na literatura, tais quais a elaboração de um manual de orçamento, a presença da comissão de orçamento, o tempo de preparação e o período.

Conforme a Quadro 1, analisa-se a presença do manual de orçamento em estudos dos autores: Schmidgall e Ninemeier (1989); Schmidgall et al., (1996); Schmidgall e DeFranco (1998); Jones (1998); Jones (2008b); Steed e Gu (2009); Uyar e Bilgin (2011); Castro et al., (2012); Codesso, Lunkes e Suave (2013); Lunkes, Codesso e Ripoll-Feliu (2014); Lunkes et al., (2014); Pereira et al., (2015); Codesso et al., (2015).

Quadro 1- Planejamento e elaboração do orçamento

\begin{tabular}{|l|l|l|l|l|l|l|l|l|l|l|l|l|l|}
\hline PRINCIPAIS QUESTÕES PESQUISADAS & & & & & & & & & & & & & \\
\\
SOBRE PRÁTICAS DE ORÇAMENTO
\end{tabular}

Revista Ambiente Contábil - ISSN 2176-9036 - UFRN - Natal-RN. v. 9. n. 2, p. 23 - 40, jul./dez. 2017 
A comissão de orçamento constitui-se de um comitê responsável por planejar, executar e controlar o funcionamento do orçamento e podem fazer parte desse grupo: proprietários, diretores, gerentes gerais, controller e outros (SCHNORRENBERGER; LUNKES; DAITX, 2014). Na literatura, os estudos de Jones (1998) e Steed e Gu (2009) evidenciaram a existência dessa comissão e quais os componentes encarregados da mesma.

Além disso, percebe-se que as pesquisas efetuadas por Lunkes et al., (2014); Pereira et al., (2015) e Codesso et al., (2015) destacaram o tempo de preparação do orçamento, com divergências temporais em cada setor empresarial estudado e também para cada empresa.

Quantos às formas de elaboração do orçamento classificam-se em top-down, bottomup ou como uma combinação entre essas duas maneiras. Orçamentos concebidos pela alta administração e repassados aos níveis inferiores são considerados top-down. Já a situação oposta, quando os diversos segmentos da empresa elaboram um orçamento e repassam para a alta administração, conceitua-se o bottom-up, o qual se caracteriza como a escolha predominante nos estudos de Codesso, Lunkes, Suave (2013), Castro et al., (2012), Schmidgall e DeFranco (1998), Schmidgall et al., (1996). Por fim, orçamentos com participação conjunta da alta administração e dos segmentos da firma denominam-se como uma combinação.

As bases para o estabelecimento da receita são responsáveis por determinar como a organização elaborará seu processo orçamentário. Na literatura, pode-se encontrar como base as influências: dados históricos baseados em percentuais de aumento (SCHMIDGALL; BORCHGREVINK; ZAHL-BEGNUM, 1996; JONES, 2008b; LUNKES et al., 2014), do proprietário (STEED; GU, 2009) ou de outros indicadores (JONES, 1998; LUNKES et al., 2014).

O tempo necessário para a elaboração do orçamento e acompanhamento de resultados denomina-se período orçamentário. Além disso, após a composição do orçamento, as revisões orçamentárias tornam-se imprescindíveis para a análise do bom funcionamento e para que se empregue as mudanças econômicas e ambientais relevantes ao orçamento. Essas revisões podem ser mensais como visto em Jones (1998), trimestrais, tal qual Schmidgall et al., (1996) e semestrais como em Pereira et al. (2015).

Despesas de capital, avaliação dos competidores, plano de marketing, funcionários em tempo integral e equivalentes, relatório dos salários da administração, pesquisa salarial, entre outros, são itens que podem integrar o pacote orçamentário (STEED; GU, 2009).

Entre os tipos de orçamento habitualmente empregados, citam-se: empresarial, flexível, de base zero (OBZ) e por atividades (ABB). Nos estudos anteriores internacionais destaca-se o OBZ como tipo orçamentário mais utilizado (SCHMIDGALL et al., 1996; JONES, 1998; JONES, 2008; PAVLATOS; PAGGIOS, 2009; UYAR; BILGIN 2011). Já no Brasil, o orçamento empresarial figura entre os mais escolhidos (CODESSO; LUNKES; SUAVE, 2013; LUNKES; CODESSO; RIPOLL-FELIU, 2014; LUNKES et al., 2014; PEREIRA et al., 2015; CODESSO et al., 2015). Além disso, o orçamento flexível apresentase presente tanto nos estudos internacionais quanto nacionais (JONES, 1998; JONES, 2008; PEREIRA et al., 2015; CODESSO et al., 2015).

Por fim, em relação ao planejamento e elaboração do orçamento, analisa-se a prática de utilização de softwares no processo orçamentário. Destaca-se o Microsoft Excel nas pesquisas de Uyar e Bilgin (2011), Schnorrenberger, Lunkes, Daitx (2014), Codesso et al., (2015), Pereira et al., (2015). Porém, Steed e Gu (2009) relatam a utilização de outros programas para elaboração do orçamento. 


\subsection{EXECUÇÃO DO ORÇAMENTO}

Durante a execução do orçamento, um conjunto de fatores pode influir nos resultados previstos. Dentre os fatores, muitos estão relacionados com o relacionamento entre a alta direção e os diversos segmentos da empresa. Nos estudos de Uyar e Bilgin (2011), Jones (2008) e Codesso et al., (2015), destacam-se que a alta direção mantém o orçamento como um segredo. Não obstante, Codesso, Lunkes, Suave (2013), Schnorrenberger, Lunkes, Daitx (2014), concordam fortemente de que uma liderança eficaz é fornecida no processo orçamentário.

Conforme a Quadro 2, identificam-se as principais questões acerca de práticas durante a execução do orçamento.

\section{Quadro 2- Execução do orçamento}

\begin{tabular}{|c|c|c|c|c|c|c|c|c|c|c|}
\hline $\begin{array}{l}\text { PRINCIPAIS QUESTÕES PESQUISADAS SOBRE PRÁTICAS } \\
\text { DE ORÇAMENTO }\end{array}$ & 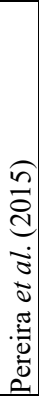 & 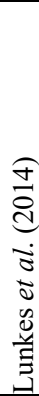 & 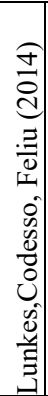 & 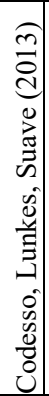 & 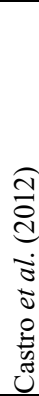 & 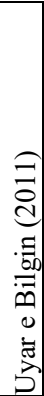 & 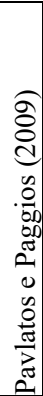 & 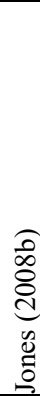 & 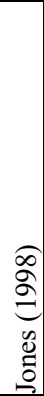 & 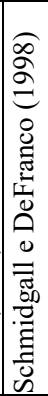 \\
\hline \multicolumn{11}{|l|}{ EXECUÇÃO DO ORÇAMENTO } \\
\hline \multicolumn{11}{|l|}{ Avaliar o processo de execução } \\
\hline \multicolumn{11}{|l|}{ Objetivos e finalidades do orçamento } \\
\hline \multicolumn{11}{|l|}{ Informações utilizadas no orçamento } \\
\hline Aspectos comportamentais relacionados ao orçamento & & & & & & & & & & \\
\hline
\end{tabular}

Fonte: Adaptado de Codesso, Lunkes, Suave (2013).

Além disso, na avaliação do processo orçamentário, identificou-se nas pesquisas de Lunkes et al., (2014), Codesso, Lunkes, Suave (2013), Uyar e Bilgin (2011), Codesso et al., (2015), Schnorrenberger, Lunkes, Daitx (2014), Jones (2008) que um bom fluxo de informações se encontra disponível para o orçamento. Por fim, como avaliação positiva, em relação às metas e objetivos, percebe-se um consenso entre os estudos, afirmando que existe coordenação entre os diversos departamentos responsáveis pelo orçamento e que as metas são fruto de um processo estruturado e alinhado com o planejamento estratégico.

Dentre os objetivos e finalidade do orçamento, destacam-se nos estudos de Jones (1998) e Pereira et al., (2015) a avaliação de desempenho. Já nas pesquisas de Pavlatos e Paggios (2009), Lunkes, Codesso, Ripoll-Feliu (2014), Codesso, Lunkes, Suave (2013), Schnorrenberger, Lunkes, Daitx (2014) o controle figura como o maior objetivo. Ainda, para Jones (2008), o orçamento apresenta-se como um auxílio para o controle das operações.

Quanto às informações utilizadas na elaboração do orçamento, Uyar e Bilgin (2011), Lunkes et al., (2014) utilizam-se majoritariamente de estatística da indústria / indicadores. Schnorrenberger, Lunkes, Daitx (2014) apontam as análises de mercado como informações mais relevantes.

Os aspectos comportamentais referentes ao orçamento coincidem em todos os estudos, os quais tiverem esse enfoque, assinalando que a participação é fundamental para os gestores aceitarem seus orçamentos e considerá-los atingíveis dentro das empresas como visto em Jones (2008), Uyar e Bilgin (2011), Lunkes, Codesso, Ripoll-Feliu (2014), Lunkes et al., 
(2014), Codesso, Lunkes, Suave (2013), Castro et al., (2012), Schnorrenberger, Lunkes, Daitx (2014), Codesso et al., (2015), Pereira et al., (2015).

\subsection{CONTROLE E AVALIAÇÃO DE DESEMPENHO}

Um dos objetivos do orçamento configura-se como o controle entre o que foi orçado e o realizado do período (UYAR; BILGIN, 2011). O Quadro 3 foca-se na avaliação de desempenho das práticas orçamentárias da organização, tais quais utilização de benchmarking e qual o papel desempenhado pelo orçamento nesse controle, ainda, aponta algumas práticas que auxiliam nesse controle.

Quadro 3 - Avaliação de desempenho

\begin{tabular}{|c|c|c|c|c|c|c|c|c|c|c|}
\hline 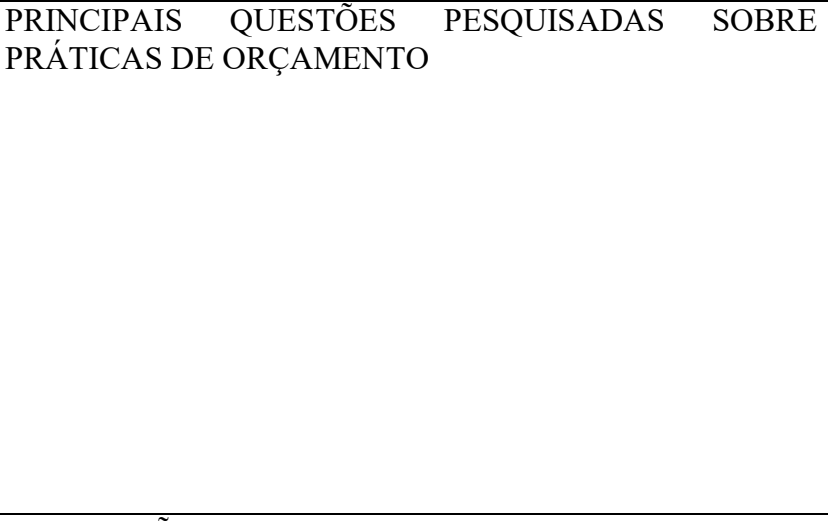 & 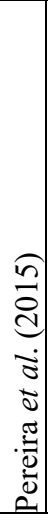 & 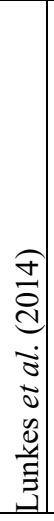 & 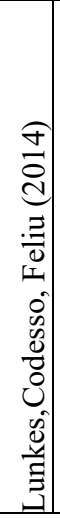 & 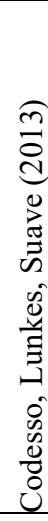 & 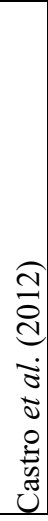 & 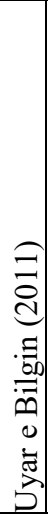 & $\begin{array}{l}\text { Oे } \\
\infty \\
0 \\
0 \\
0 \\
0 \\
0 \\
0 \\
0\end{array}$ & 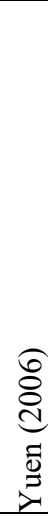 & 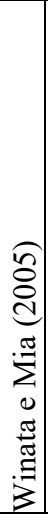 & 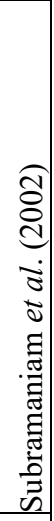 \\
\hline \multicolumn{11}{|l|}{$\underline{\text { AVALIACCÃO DE DESEMPENHO }}$} \\
\hline \multicolumn{11}{|l|}{ Utilização de benchmarking } \\
\hline \multicolumn{11}{|l|}{ Papel desempenhado pelo orçamento no monitoramento } \\
\hline \multicolumn{11}{|l|}{ Principais medidas de desempenho utilizadas } \\
\hline \multicolumn{11}{|l|}{ Responsáveis pela análise das variações } \\
\hline \multicolumn{11}{|l|}{ Finalidades da avaliação das variações do orçamento } \\
\hline \multicolumn{11}{|l|}{ OUTROS TEMAS } \\
\hline \multicolumn{11}{|l|}{ Avaliar a satisfação dos empregados em relação ao orçamento } \\
\hline \multicolumn{11}{|l|}{ Relação entre participação no orçamento e uso de TI } \\
\hline Descentralização e participação no orçamento & & & & & & & & & & \\
\hline
\end{tabular}

Fonte: Adaptado de Codesso, Lunkes, Suave (2013).

A prática de benchmarking, juntamente com a utilização de pontos de referência aparece nos estudos de Jones (2008), Uyar e Bilgin (2011) e outros. Ainda, Uyar e Bilgin (2011) aponta o resultado de anos anteriores como principal medida de desempenho, diferentemente de Lunkes et al., (2014) que considera os valores orçados como mais relevantes.

A literatura também mostra que, quanto ao papel do orçamento no monitoramento, pode-se considerá-lo como o principal indicador de desempenho e que os principais responsáveis pela análise de variações em relatórios orçamentários os altos gerentes, diretores e gerentes de departamento.

Como finalidade de avaliação das variações de orçamento Uyar e Bilgin (2011) destaca o controle de custos e gastos. Os estudos de Codesso, Lunkes, Suave (2013), Lunkes et al., (2014), Schnorrenberger, Lunkes, Daitx (2014), Pereira et al., (2015). Lunkes, Codesso, 
Ripoll-Feliu (2014) também incluem a identificação de um problema potencial a tempo de minimizar seu impacto, essa finalidade também figura nos estudos de Codesso, Lunkes, Suave (2013) e Lunkes et al., (2014).

\section{PROCEDIMENTOS METODOLÓGICOS}

Quanto aos objetivos, a presente pesquisa é descritiva, com abordagem quantitativa. $\mathrm{Na}$ concepção de Gil (2010), a pesquisa descritiva tem como principal objetivo descrever características de determinadas populações ou fenômeno ou o estabelecimento de relações entre as variáveis. Uma de suas características mais significativas é a utilização de técnicas padronizadas de coletas de dados. Portanto, descrever significa identificar, relatar, comparar, entre outros aspectos (BEUREN, 2014).

Já quanto aos procedimentos de pesquisa, foi utilizado o levantamento ou survey, que na concepção de Gil (2010), se caracteriza basicamente, pela solicitação de informações a um grupo significativo de pessoas acerca do problema estudado para em seguida, mediante análise quantitativa, obter as conclusões correspondentes aos dados coletados. Segundo Beuren (2014), as pesquisas de levantamento normalmente são mais bem utilizadas em estudos descritivos, cujos resultados não há grande exigência em aprofundar.

Além disso, a presente pesquisa possui abordagem quantitativa, por se caracterizar pelo emprego de instrumentos estatísticos, tanto na coleta quanto no tratamento dos dados (BEUREN, 2014). Esse tipo de estudo deve ser realizado quando o pesquisador deseja obter melhor entendimento do comportamento de diversos fatores e elementos que influem sobre determinado fenômeno (RICHARDSON, 1999).

Para a coleta de dados, utilizou-se questionário elaborado a partir das principais características apontadas na revisão de literatura (DAITX \& SCHNORRENBERGER, 2014; CASTRO et al., 2012; JONES, 1998, 2008A, 2008B; PAVLATOS \& PAGGIOS, 2008; SCHMIDGALL, BORCHGREVINK \& ZAHL-BEGNUM, 1996; SCHMIDGALL \& DEFRANCO, 1998; SCHMIDGALL \& NINEMEIER, 1989, 1986, 1987; STEED \& GU, 2009; SUBRAMANIAM, MCMANUS \& MIA, 2002; UYAR \& BILGIN, 2011; WINATA \& MIA, 2005; YUEN, 2006).

O questionário está organizado em quatro etapas: na primeira parte, os dados iniciais da companhia e dos respondentes, a segunda parte refere-se ao planejamento do orçamento, a terceira parte está relacionada à execução do orçamento e a quarta e ultima parte está relacionada à avaliação de desempenho.

\subsection{POPULAÇÃO E AMOSTRA E COLETA DE DADOS DO ESTUDO}

Com relação à população e amostra, o questionário eletrônico foi enviado para 342 grupos econômicos que representam 650 concessionárias no Estado de Santa Catarina, sendo utilizado para envio, o Software mail2easy de gestão de e-mail. Foram disparados os primeiros e-mails em 14 de setembro de 2015 e semanalmente foram reenviados e-mails reforçando a importância da pesquisa para uma melhor compreensão do tema e sua utilização e utilidade percebida pelo setor. O último reenvio foi feito em 04/10/2015. Foram consideradas as respostas obtidas até o dia 15/11/2015. No total, foram disparados $1328 e$ mails, dos quais 355 foram visualizados, 55 efetivamente acessados e 20 respondidos e retornados, correspondendo a 5,84\% do total de amostra. Os nomes dos respondentes foram omitidos para preservar o seu anonimato.

Os dados coletados foram dispostos em tabelas, sendo analisada a quantidade de respostas de cada questão. Para as questões relativas ao planejamento do orçamento e avaliação de desempenho, foram identificadas as quantidades percentuais de respostas de cada 
questão e comparadas com os principais achados na literatura que trataram do tema. Nas questões relativas à execução do orçamento foi utilizada a escala Likert de 5 pontos, para uma melhor análise dos resultados, sendo estabelecido um Ranking Médio (RM)) para mensurar o grau de concordância dos sujeitos que responderam aos questionários (UYAR E BILGIN, 2011). Realizou-se a verificação quanto à concordância ou discordância das questões avaliadas, através da obtenção do RM da pontuação atribuída às respostas, relacionando-os à frequência das respostas dos respondentes que fizeram tal atribuição, onde os valores menores que 3 são considerados como discordantes e, maiores que 3 , como concordantes. $\mathrm{O}$ valor exatamente 3 é considerado "indiferente" ou "sem opinião", sendo o "ponto neutro", equivalente aos casos em que os respondentes deixaram em branco.

\section{ANÁLISE E DISCUSSÃO DOS RESULTADOS}

Preliminarmente o questionário traz características gerais das empresas, visando conhecer o perfil das organizações. Dentre as primeiras constatações destaca-se o fato da maioria das empresas, ou seja, 11 afirmarem possuir processo formal de orçamento e ter profissionais responsáveis com idade inferior a 50 anos. Destes, 4 trabalham há menos de 5 anos na área, 2 entre 5 e 10 anos e 4 há mais de 10 anos, sendo que 1 respondente não forneceu a informação. Destaque também para a qualificação, pois, 19 dos 20 pesquisados possuem formação mínima em nível de graduação, sendo que 7 tem graduação, 11 pósgraduação e 1 doutorado. Constata-se também que $70 \%$ das empresas faturam acima de 60 milhões de reais por ano e $10 \%$ faturam entre 10 milhões e 60 milhões de reais. Quanto ao número de empregados, $45 \%$ empregam até 100 pessoas e $45 \%$ entre 100 e 500 pessoas.

\subsection{PLANEJAMENTO DO ORÇAMENTO}

Das companhias pesquisadas, $55 \%$ afirmaram ter processo orçamentário formal. Na medida em que aumentam de porte e complexidade, passam a adotar, gradativamente, procedimentos comuns às organizações de grande porte, no caso o Planejamento Orçamentário, o que se confirma na pesquisa realizada, onde 9 das 11 empresas que possuem orçamento faturam acima de $\mathrm{R} \$ 60$ milhões de reais anuais

Mais da metade possui manual de orçamento. Estes resultados destoam dos encontrados por Castro et al., (2012) que constataram que 100\% das empresas possuíam manual. Entretanto se aproximam a outros estudos, como o de Uyar e Bilgin (2011) e Daitx e Schnorrenberger (2014). No que se refere a comissão de orçamento, 63,6\% disseram possuir, mesmo resultado de Daitx e Schnorrenberger (2014), enquanto 79\% foi o resultado encontrado por Castro et al., (2012), 87\% por Uyar e Bilgin (2011). Quanto aos componentes da comissão de orçamento, constatou-se que o Gerente de Contabilidade/Controller é integrante em 90,9\% das empresas, seguido pelo Gerente Geral/Diretor da empresa em 81,8\% e Gerente Financeiro/Tesoureiro em 63,6\%. Os Proprietários participam das comissões em $54,5 \%$. Não foram mencionados no questionário o Gerente de Marketing e o Diretor de Orçamento/Manager. No caso do Diretor de Orçamento, muito provavelmente, por não haver esse cargo nas empresas analisadas. De qualquer forma a pesquisa mostra a grande participação do Gerente de Contabilidade e Controller na elaboração do orçamento. Ao analisar os resultados quanto ao planejamento do orçamento, contata-se que $45,5 \%$ necessitam de 41 a 80 horas para preparar e revisar o orçamento. As que necessitam Menos de 40 horas, de 81 a 120 horas ou mais de 120 horas, ficaram em 18,2\% para cada item. Estes resultados diferem dos achados de Castro et al., (2012) e de Uyar e Bilgin (2011).

Com relação à participação no processo orçamentário, constata-se que $54,5 \%$ das empresas possuem processo participativo no que se refere a elaboração do orçamento, estes 
dados se aproximam dos estudos de Uyar e Bilgin (2011) que encontraram 42\% de elaboração conjunta na formação do orçamento. Já o modelo bottom-up com 18,2\% e top-down com $27,3 \%$ compõe os demais processos de elaboração do orçamento. Por outro lado, se distanciam dos achados de Castro et al., (2012), W que aponta a elaboração conjunta como a menos utilizada pelas organizações pesquisadas, com $14 \%$. Já a pesquisa de Daitx e Schnorrenberger (2014) apresentou que 100\% das empresas utilizam a elaboração conjunta.

O horizonte do tempo planejado para $81,8 \%$ das empresas é de 1 ano e em $18,2 \%$ dos casos o horizonte é de apenas 1 mês. Esse resultado se aproxima dos achados de Uyar e Bilgin (2011) na Turquia com 74\%, de Castro et al., (2012) no Brasil com 79\% e Daitx e Schnorrenberger (2014) também no Brasil com 75\%.

Além disso, o orçamento sofre algum tipo de revisão durante o ano. No entanto, pelos achados, essa revisão não possui um padrão definido, pois se dá em $36,4 \%$ das vezes de forma semestral, sendo 18,2\% mensal e 9,1\% Trimestralmente. Em 45,5\% das vezes, as empresas utilizam outras sistemáticas de revisão do orçamento. Esses achados se aproximam da pesquisa de Castro et al. (2012) e Daitx e Schnorrenberger (2014).

Quanto à periodicidade dos relatórios orçamentários, 90,9\% são mensais. Esse resultado se aproxima das pesquisas de Uyar e Bilgin (2011), Castro et al., (2012) e Daitx e Schnorrenberger (2014) que constataram que 92\%, 79\% e $83 \%$ das empresas turcas e brasileiras, respectivamente, geram relatórios mensais.

Quanto à base para estabelecimento do aumento da receita, 63,6\% das empresas afirmam que o fazem baseado na inflação prevista, 54,5\% utilizam também percentual de aumento em relação ao ano passado. Apenas $27,3 \%$ afirmaram utilizar também a base na melhoria do conjunto competitivo e método estatístico (regressão etc.). Esses resultados se aproximam dos estudos de Schmidgall et al., (1996) e Jones (2008a) que apontam que na definição do orçamento anual, entre as informações mais comumente utilizadas estão os dados do ano anterior (dados operacionais históricos).

Dos itens que fazem parte do pacote orçamentário, 63,6\% das empresas possuem além das informações financeiras das operações, o plano de marketing e também funcionários em tempo integral ou equivalentes. 54,5\% utilizam o item Despesas de Capital e 27,3\% também utilizam avaliação de competidores e relatórios de salários da administração. Steed e $\mathrm{Gu}$ (2009) demonstraram que as despesas de capital foram apontadas por $87,8 \%$, avaliação da competição por $82,9 \%$, plano de marketing por $80,5 \%$, funcionários em tempo integral e equivalentes por $70,7 \%$, salários da administração por $68,3 \%$, pesquisa salarial por $36,6 \%$ e outros com $17,1 \%$.

As empresas analisadas informaram fazer alterações decorrentes do volume de vendas em $63,6 \%$ da amostra, o que demonstram utilizarem um orçamento flexível. Contudo $81,8 \%$ das empresas informaram utilizar o orçamento empresarial e apenas 18,2\% afirmaram utilizar o orçamento flexível. Estes achados destoam dos outros estudos que encontraram a utilização de diferentes tipos como o OBZ, (JONES, 1998) no Reino Unido e (PAVLATOS; PAGGIOS, 2009) na Grécia, Flexível (UYAR; BILGIN, 2011) na Turquia, entre outros.

O Software Microsoft Excel é utilizado por 91\% das empresas para elaboração do processo orçamentário, e 45,5\% afirmaram utilizar também outras tecnologias. Steed e Gu (2009) nos EUA e Uyar e Bilgin (2011) na Turquia, também encontraram altos índices de utilização de planilhas, sendo $71,4 \%$ e $78,5 \%$, respectivamente. Portanto, é possível a realização do orçamento com a utilização dessa ferramenta que acarreta baixo investimento em sistemas para a elaboração além de maior flexibilidade. Mas, a utilização dessa ferramenta poderá ser menos eficaz quando houver grande volume de dados a processar.

Verificou-se ainda, que $63,6 \%$ das empresas elaboram e aplicam o planejamento estratégico e $27,3 \%$ não elaboram. Apenas $9,1 \%$ das empresas trabalham com horizonte superior a 5 anos, o que difere das pesquisas Schmidgall e Ninemeier (1989), nos EUA, 
Schmidgall et al., (1996), na Escandinávia e Schmidgall et al., (1996), nos EUA, com respectivos $33,3 \%, 29,6 \%$ e $76 \%$ das companhias possuindo este horizonte de tempo para o planejamento.

\subsection{EXECUÇÃO DO ORÇAMENTO}

Os Resultados encontram-se na Tabela 1 e foram obtidos com o uso da escala Likert sendo que 1 significa "discordo totalmente" e 5, "concordo totalmente".

Tabela 1: Percepção da execução do processo orçamentário

\begin{tabular}{|c|c|c|c|c|c|c|c|}
\hline Item & 1 & 2 & 3 & 4 & 5 & $\mathbf{R M}$ & DP \\
\hline Orçamento é considerado um segredo da alta direção & 5 & 3 & 1 & 2 & 0 & 2,00 & 1,18 \\
\hline Uma liderança eficaz é fornecida no processo orçamentário & 0 & 0 & 2 & 5 & 4 & 4,18 & 0,75 \\
\hline Um bom fluxo de informações está disponível para o orçamento & 0 & 0 & 2 & 4 & 5 & 4,27 & 0,79 \\
\hline Coordenação entre as áreas é conseguida facilmente & 0 & 4 & 0 & 4 & 3 & 3,55 & 1,29 \\
\hline $\begin{array}{l}\text { Metas são definidas primeiro nas áreas e depois submetidas à } \\
\text { alta direção }\end{array}$ & 2 & 3 & 1 & 4 & 1 & 2,91 & 1,38 \\
\hline $\begin{array}{l}\text { Metas são definidas pela alta direção e depois são coletadas } \\
\text { opiniões nas áreas }\end{array}$ & 0 & 3 & 1 & 3 & & 3,73 & 1,27 \\
\hline $\begin{array}{l}\text { Metas emergem de um processo estruturado e alinhado com o } \\
\text { Planej. Estratégico }\end{array}$ & 0 & 2 & 0 & 4 & 5 & 4,09 & 1,14 \\
\hline
\end{tabular}

Fonte: Dados da Pesquisa

Com relação à percepção da execução do orçamento, o item que trata do orçamento ser considerado um segredo da alta direção, apresenta alto grau de discordância (Ranking Médio 2,00), o que difere das pesquisas de Uyar e Bilgin (2011) onde se tem um RM de 3,78. Outro ponto que difere da pesquisa de Uyar e Bilgin (2011) é o item que trata sobre se as metas são definidas primeiro nas áreas e depois submetidas à alta direção, o que apresentou um RM de 2,91, em comparação com a pesquisa de Uyar e Bilgin (2011) que apresentou um RM de 3,67. Esse resultado difere da informação de que em mais de $54,5 \%$ das empresas o orçamento é participativo. Outro ponto de divergência é em relação ao item coordenação entre as áreas como facilitador do processo orçamentário, o que no caso das concessionárias possui um RM de 3,55 e nas pesquisas de Uyar e Bilgin (2011) está acima de 4,00. Os demais resultados estão de acordo com as pesquisas de Uyar e Bilgin (2011).

A tabela 2 apresenta os objetivos pretendidos com a elaboração do orçamento. Pode-se perceber um alto grau de concordância com relação a maioria dos itens pesquisados, com destaque para o objetivo de planificar, controlar e valorar os resultados, onde $100 \%$ dos respondentes concordaram completamente. $\mathrm{O}$ item com menor grau de concordância foi o relacionado com o objetivo de criar incentivos e recompensas aos empregados, onde as respostas foram bem divididas, o que caracteriza que parte das concessionárias pesquisadas não têm um plano de remuneração variável baseado no desempenho do orçamento. 
Myrna Modolon Lima, José Luiz Vailatti, Rogério João Lunkes, Valdirene Gasparetto e Darci Schnorrenberger

Tabela 2: Os objetivos pretendidos com a elaboração do orçamento

\begin{tabular}{|c|c|c|c|c|c|c|c|}
\hline Item & 1 & 2 & 3 & 4 & 5 & $\mathbf{R M}$ & DP \\
\hline Previsões de futuro & 0 & 0 & 2 & 3 & 6 & 4,36 & 0,81 \\
\hline Maximizar os resultados & 0 & 0 & 0 & 2 & 9 & 4,82 & 0,40 \\
\hline Inserção de novos produtos e serviços na empresa & 0 & 0 & 4 & 2 & 5 & 4,09 & 0,94 \\
\hline Planificar, controlar e valorar os resultados & 0 & 0 & 0 & 0 & 11 & 5,00 & 0,00 \\
\hline Criar incentivos e recompensas aos empregados & 1 & 2 & 1 & 2 & 5 & 3,73 & 1,49 \\
\hline Decisões de preço & 0 & 1 & 2 & 3 & 5 & 4,09 & 1,04 \\
\hline Controle & 0 & 0 & 0 & 2 & 9 & 4,82 & 0,40 \\
\hline Auxiliar no planejamento de longo prazo & 0 & 1 & 1 & 0 & 9 & 4,55 & 1,04 \\
\hline Coordenar as operações & 0 & 0 & 0 & 3 & 8 & 4,73 & 0,47 \\
\hline Auxiliar no planejamento de curto prazo & 0 & 1 & 0 & 3 & 7 & 4,45 & 0,93 \\
\hline Avaliar o desempenho & 1 & 0 & 0 & 1 & 9 & 4,55 & 1,21 \\
\hline Motivar os gerentes & 0 & 1 & 1 & 3 & 6 & 4,27 & 1,01 \\
\hline Motivar os empregados & 0 & 1 & 1 & 4 & 5 & 4,18 & 0,98 \\
\hline Comunicar os planos & 0 & 0 & 0 & 5 & 6 & 4,55 & 0,52 \\
\hline Planejar a rentabilidade & 0 & 0 & 0 & 3 & 8 & 4,73 & 0,47 \\
\hline
\end{tabular}

Fonte: Dados da Pesquisa.

Na Tabela 3 verifica-se as fontes utilizadas pelas empresas no processo orçamentário. Os dados foram coletados com auxílio de uma escala Likert, onde 1 (Nunca), e 5 (Sempre).

Tabela 3 - Informações utilizadas na elaboração do orçamento

\begin{tabular}{|c|c|c|c|c|c|c|c|}
\hline Item & 1 & 2 & 3 & 4 & 5 & $\mathbf{R M}$ & DP \\
\hline Resultado dos anos anteriores - realizados & 0 & 0 & 0 & 3 & 8 & 4,73 & 0,47 \\
\hline Estatística da indústria / indicadores & 0 & 0 & 1 & 4 & 6 & 4,45 & 0,69 \\
\hline Análises de mercado & 0 & 0 & 1 & 3 & 7 & 4,55 & 0,69 \\
\hline Indicadores econômicos nacionais & 0 & 0 & 0 & 5 & 6 & 4,55 & 0,52 \\
\hline Números de anos anteriores & 0 & 0 & 0 & 4 & 7 & 4,64 & 0,50 \\
\hline Indicadores econômicos locais ou regionais & 0 & 0 & 0 & 5 & 6 & 4,55 & 0,52 \\
\hline
\end{tabular}

Fonte: Dados da Pesquisa

Os valores médios superiores a 4,00 mostram que todos os indicadores listados são utilizados na definição de orçamentos (Tabela 3). No entanto, os indicadores mais utilizados são resultados de anos anteriores realizados (Ranking Médio $=4,73$ e Desvio Padrão $=0,22$ ) e números de anos anteriores (Ranking Médio $=4,64$ e Desvio Padrão $=0,25$ ). Os dados da tabela 3 estão de acordo com os resultados apresentados pela pesquisa de Uyar e Bilgin (2011). O próprio segmento requer a utilização de várias fontes de informação tendo em vista a própria característica do negócio. A estatística da indústria, por exemplo, pode ser entendida aqui como os dados fornecidos pela Montadora às concessionárias no que diz respeito às informações de expectativa de produção de veículos. Nesse sentido é importante para a Montadora adequar a sua produção à demanda, sendo que essa análise é utilizada pelas concessionárias como objetivos comerciais a serem cumpridos junto à Montadora. 


\subsection{CONTROLE E AVALIAÇÃO DE DESEMPENHO}

O orçamento possui como objetivo o controle e a avaliação desempenho, que é obtido quando se compara o previsto e realizado. Esse processo permite identificar possíveis falhas decorrentes do planejamento. No Quadro 4 apresenta-se o resultado da pesquisa sobre controle e avaliação de desempenho.

Quadro 4 - Controle e Avaliação de Desempenho

\begin{tabular}{|c|c|c|c|c|c|c|c|c|}
\hline \multirow{3}{*}{\multicolumn{2}{|c|}{ 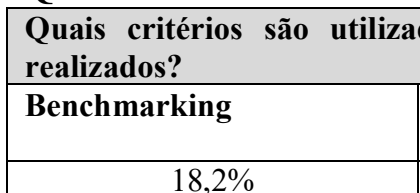 }} & \multirow{2}{*}{\multicolumn{2}{|c|}{$\begin{array}{l}\text { dos para avaliar difere } \\
\begin{array}{l}\text { Não usam pontos de } \\
\text { referência }\end{array} \\
\end{array}$}} & \multicolumn{5}{|c|}{ significativas entre os valores orçados e } \\
\hline & & & & \begin{tabular}{l|l} 
Usa \\
refe
\end{tabular} & $\begin{array}{l}\text { m por } \\
\text { rência }\end{array}$ & itos de & Outro & \\
\hline & & & & & $63,6^{6}$ & & & $0 \%$ \\
\hline \multicolumn{9}{|c|}{ Caso sim, qual(is) a(s) forma(s) utilizada(s)? } \\
\hline \multicolumn{2}{|c|}{ Valor monetário } & \multicolumn{2}{|c|}{ Porcentagem } & \multicolumn{3}{|c|}{$\begin{array}{l}\text { Repetição de uma } \\
\text { variação adversa }\end{array}$} & \multicolumn{2}{|c|}{ Outro } \\
\hline \multicolumn{2}{|c|}{$36,4 \%$} & \multicolumn{2}{|c|}{$81,8 \%$} & \multicolumn{3}{|c|}{$0 \%$} & \multicolumn{2}{|r|}{$9,1 \%$} \\
\hline \multirow{2}{*}{\multicolumn{2}{|c|}{$\begin{array}{l}\text { Qual o papel do orçamento } \\
\text { Principal indicador de } \\
\text { desempenho }\end{array}$}} & monitora & mento do & mpe & real da & rganização? & & \\
\hline & & \multicolumn{2}{|c|}{$\begin{array}{l}\text { Um dos poucos } \\
\text { indicadores-chave } \\
\text { utilizados na } \\
\text { organização }\end{array}$} & \multicolumn{3}{|c|}{$\begin{array}{l}\text { Pouco utilizado como } \\
\text { medida de desempenho }\end{array}$} & \multicolumn{2}{|c|}{ Outro } \\
\hline \multicolumn{2}{|c|}{$54,5 \%$} & & \multicolumn{3}{|c|}{$9,1 \%$} & \multicolumn{2}{|r|}{$9,1 \%$} \\
\hline \multicolumn{9}{|c|}{ Quais são as principais medidas de desempenho utilizadas? } \\
\hline \multirow{2}{*}{$\begin{array}{l}\begin{array}{l}\text { Resultados } \\
\text { de anos } \\
\text { anteriores }\end{array} \\
72,7 \%\end{array}$} & $\begin{array}{l}\text { Valores } \\
\text { orçados }\end{array}$ & \multicolumn{2}{|c|}{\begin{tabular}{|lr|}
\multicolumn{2}{|l|}{ Indicadores e metas } \\
do & Balanced \\
scorecard & \\
\end{tabular}} & \multicolumn{2}{|c|}{$\begin{array}{l}\text { Comparações } \\
\text { com os } \\
\text { concorrentes }\end{array}$} & \multicolumn{2}{|c|}{$\begin{array}{l}\text { Estatísticas do } \\
\text { setor }\end{array}$} & Outros \\
\hline & $72,7 \%$ & \multicolumn{2}{|c|}{$9,1 \%$} & \multicolumn{2}{|c|}{$27,3 \%$} & \multicolumn{2}{|l|}{$45,5 \%$} & $0 \%$ \\
\hline \multicolumn{9}{|c|}{ Quem são os responsáveis pela análise das variações? } \\
\hline $\begin{array}{l}\text { Alta } \\
\text { Direção }\end{array}$ & $\begin{array}{l}\text { Gerente da } \\
\text { empresa }\end{array}$ & \multicolumn{2}{|c|}{$\begin{array}{ll}\text { Chefes } & \text { de } \\
\text { Departamento } & \end{array}$} & $\begin{array}{l}\text { Comiss } \\
\text { Orçam }\end{array}$ & $\begin{array}{ll}\text { ão } & \text { de } \\
\text { ento } & \end{array}$ & Controller & & Outros \\
\hline $81,8 \%$ & $63,6 \%$ & 27 , & $3 \%$ & & $3 \%$ & $54,5 \%$ & & $0^{\circ}$ \\
\hline A análise da & variação aconte & & & & & & & \\
\hline Mensalment & Trimes & tralmente & Semestraln & nente & Anualm & ente & Out & \\
\hline $90,9 \%$ & & $0 \%$ & $9,1 \%$ & & &, $1 \%$ & & $0 \%$ \\
\hline Quais as fin: & lidades da an & ise das varia & es do or & nento? & & & & \\
\hline $\begin{array}{l}\text { Avaliar o } \\
\text { desempenh } \\
\text { o do } \\
\text { gerente }\end{array}$ & $\begin{array}{l}\text { Avaliar a } \\
\text { capacidade } \\
\text { de previsão } \\
\text { do gerente }\end{array}$ & $\begin{array}{l}\text { Controlar } \\
\text { custos/des }\end{array}$ & $\begin{array}{ll}\text { pesas } & \text { os } \\
\end{array}$ & $\begin{array}{l}\text { Identifi } \\
\text { problen } \\
\text { potenci } \\
\text { tempo } \\
\text { lo } \\
\end{array}$ & $\begin{array}{l}\text { car um } \\
\text { na } \\
\text { al a } \\
\text { de evitá- }\end{array}$ & $\begin{array}{l}\text { Fornecer } \\
\text { feedback p } \\
\text { o orçamento } \\
\text { próximo } \\
\text { período } \\
\end{array}$ & \begin{tabular}{l|} 
para \\
o do \\
\end{tabular} & Outros \\
\hline $63,6 \%$ & $18,2 \%$ & 90 , & $9 \%$ & & $5 \%$ & $54,5 \%$ & & $0 \%$ \\
\hline No controle & rçamentário, q & ais etapas & ão aplicac & & & & & \\
\hline $\begin{array}{l}\text { Análise } \\
\text { comparativa }\end{array}$ & $\begin{array}{l}\text { Identif } \\
\text { desvios }\end{array}$ & icação dos & $\begin{array}{l}\text { Análise } \\
\text { causas } \\
\text { desvios }\end{array}$ & $\begin{array}{l}\text { das } \\
\text { dos }\end{array}$ & $\begin{array}{l}\text { Medidas } \\
\text { adequaç }\end{array}$ & de & Out & ros \\
\hline $90,9 \%$ & & $4,5 \%$ & $36,4^{\circ}$ & & & $4,5 \%$ & & $0 \%$ \\
\hline
\end{tabular}

Fonte: Dados da pesquisa.

No resultado da pesquisa, conforme quadro 4, foi observado que $63,6 \%$ dos respondentes, utilizam pontos de referências como critérios para avaliar diferenças significativas entre os valores orçados e realizados. Além disto, $81,8 \%$ das empresas afirmaram utilizar porcentagem como forma de apresentar as variações no orçamento e 36,6\% também utilizam valor monetário. Esses resultados estão de acordo com os achados de Pereira 
et al., (2015), Codesso, Lunkes, Suave (2013), Castro et al., (2012), mas divergem da pesquisa de Daitx e Schnorrenberger (2014) onde mostrou que 83\% utilizam o Benchmarking.

Para $54,5 \%$ das empresas, o orçamento é o principal indicador de desempenho no papel de monitoramento do desempenho real da organização, sendo também um dos poucos indicadores chaves utilizados pela organização para $36,4 \%$ das empresas pesquisadas, o que diverge um pouco das pesquisas de Castro et al., (2012), Codesso, Lunkes, Suave (2013), Daitx e Schnorrenberger (2014) e Pereira et al., (2015) que apresentaram resultados acima de $75 \%$. Esse resultado pode ser explicado pelo fato das concessionárias possuírem diversos indicadores além do orçamento e que são fornecidos pelas próprias montadoras.

Os resultados de anos anteriores e também os valores orçados são considerados por $72,7 \%$ das empresas como as principais medidas de desempenho utilizadas. Esse resultado corrobora com as pesquisas de Castro et al., (2012), Codesso, Lunkes, Suave (2013).

A alta direção é responsável pela análise das variações em $81,8 \%$ das empresas pesquisadas. No entanto, os gerentes da empresa, com $63,6 \%$ e o Controller, com $54,5 \%$ também figuram como responsáveis pela análise das variações. Os resultados apresentados estão de acordo com as pesquisas de Codesso, Lunkes, Suave (2013) e Pereira et al., (2015), onde apresentaram o resultado de $83 \%$ e $75 \%$ para a alta direção como responsável pela análise das variações, além dos gerentes e chefes de departamentos com altos índices de participação.

A análise da variação do orçamento acontece de forma mensal em $91 \%$ das empresas, tendo em vista que a finalidade principal das análises da variação é para controle de custos /despesas, identificar um problema potencial a tempo de evitá-lo e para fornecer feedback para o orçamento do próximo período, para $54,5 \%$ dos respondentes. Já a análise comparativa é utilizada por aproximadamente $91 \%$ das empresas, mas podendo ser destacado também a identificação de desvios e as medidas de adequação, por 54,5\% das empresas pesquisadas que utilizam orçamento.

\section{CONSIDERAÇÕES FINAIS}

O presente estudo teve por objetivo analisar as principais práticas de orçamentos das concessionárias de veículos de Santa Catarina. Para alcance dos objetivos, utilizou-se pesquisa descritiva, com abordagem quantitativa, onde o problema foi tratado por meio da utilização de um questionário que foi elaborado a partir das principais características apontadas na literatura na elaboração, execução e controle do orçamento.

A maioria das empresas pesquisadas demonstrou utilizar um processo formal de orçamento, principalmente nas concessionárias que possuem gestores com maior nível de qualificação. Pelo nível de exigência imposto pelas Montadoras para a concessão da marca, onde são estabelecidos objetivos e metas que devem ser perseguidos pelas concessionárias, é possível perceber um maior nível de organização por parte das empresas pesquisadas, tendo em vista demonstrarem possuir manual de orçamento e comitês específicos para tratar do orçamento.

Ficou evidente na pesquisa, que o orçamento participativo é o mais utilizado pelas concessionárias. Esse fator pode ser explicado pelo fato de que as concessionárias possuírem estruturas de centros de resultados, os quais possuem gerentes responsáveis. Isso permite que eles possam participar do processo orçamentário, desde o seu planejamento até a sua execução e controle.

Os relatórios orçamentários são ferramentas utilizadas por quase todas as concessionárias de forma mensal, onde são feitos os comparativos entre receitas e despesas orçadas e realizadas. As métricas utilizadas para definição das receitas são a inflação prevista e percentual de aumento da receita. Contudo, como característica do ramo, esses percentuais 
de aumento são definidos em conjunto com a Montadora que define o plano de vendas das concessionárias e que são revisados periodicamente. Essa revisão pode ser identificada através da utilização do orçamento flexível pela maioria das concessionárias, que também utilizam o Orçamento Empresarial como ferramenta.

Quanto a execução do orçamento, as empresas, em sua maioria, discordam de que o orçamento seja considerado um segredo da alta direção e concordam que uma liderança eficaz é fornecida no processo orçamentário, bem como um bom fluxo de informações está disponível para o orçamento. A coordenação entre os departamentos pode ser apontada como fator importante, contudo na pesquisa realizada não houve concordância entre todas as concessionárias, assim como em relação às metas que são definidas primeiramente pela alta direção e depois submetidas às áreas, de acordo com a pesquisa realizada. Como principal objetivo pretendido para a elaboração do orçamento está a necessidade de planificar, controlar e valorar os resultados pretendidos, além de ajudar a avaliar o desempenho da empresa.

Os resultados apresentados mostram que os resultados dos anos anteriores, correspondem a forma de avaliação do orçamento previsto, bem como também utiliza-se análise de mercado para acompanhar a execução do orçamento nas concessionárias de veículos. As concessionárias de veículos de maneira geral concordaram que a autoridade e responsabilidade individual do gerente devem ser claras para o controle orçamentário ser eficaz, bem como a participação dos gestores é fundamental para que aceitem seus orçamentos e os tornem eficazes no processo de gestão.

As concessionárias analisadas utilizam de maneira geral pontos de referência como critérios de para avaliação de diferenças significativas entre o previsto através da utilização da porcentagem. O orçamento ainda é principal indicador de desempenho na maioria das concessionárias, sendo que os valores orçados e também resultados de anos anteriores são as principais medidas utilizadas pelas concessionárias e que são analisadas na maioria das concessionárias pela alta direção e pelos gerentes das empresas de forma mensal. As análises efetuadas têm por finalidade principal, o controle de custos e despesas, bem como avaliar o desempenho dos gerentes, através de uma análise comparativa.

Dentre as principais limitações do estudo pode-se destacar a amostra restrita, associada à forma de obtenção dos dados, que não permitiu a interação efetiva com os respondentes visando esclarecer ou ampliar a compreensão de certos pontos obscuros ou contraditórios. Naturalmente, os achados deste estudo possuem validade principalmente para a realidade das empresas investigadas.

Para estudos futuros recomenda-se a realização de investigações semelhantes em outras regiões do país e também, em outros segmentos ou setores da economia, visando identificar similaridades ou discrepâncias no trato deste tema.

\section{REFERÊNCIAS}

BEUREN, I. M. Como elaborar trabalhos monográficos em contabilidade: teoria e prática. $1^{\text {a }}$. ed. São Paulo: Atlas, 2014.

CASTRO, J. K.; SANTOS, G. R.; SOUZA, P.; LUNKES, R. J. Práticas orçamentárias aplicadas em empresas hoteleiras no Brasil: um estudo na cidade de Florianópolis. Revista Estudios y Perspectivas em Turismo, v. 21, n. 4, p. 904-924, 2012.

CODESSO, M. M.; LUNKES, R.J.; SUAVE, R. Práticas Orçamentárias Aplicadas em Empresas Hoteleiras no Brasil: um Estudo na Cidade de Balneário Camboriú. Turismo: Visão e Ação (Online), v. 15, p. 293-308, 2013. 
CODESSO, M. M. ; LYRIO, M. V. L. ; LUNKES, R. J.; PINTO, H. M. Budgeting practices applied to supermarket in the State of Santa Catarina, Brazil. Business Management Review (BMR), v. 4, p. 585-595, 2015.

DAITX, R. R. ; SCHNORRENBERGER, D. . Práticas de orçamento em empresas cerâmicas do Sul do Brasil. In: XXI CONGRESSO BRASILEIRO DE CUSTOS, 2014, NATAL. Gestão de Custos no Brasil Pós-Copa 2014 e Pré-Olimpíadas 2016, 2014.

FREZATTI, F. Orçamento empresarial: planejamento e controle gerencial. 5. ed.São Paulo: Atlas, 2009.

FREZATTI, F.; AGUIAR, A. B. D.; REZENDE, A. J. Respostas estratégicas às pressões institucionais e sucesso no atingir metas no orçamento: um estudo em uma empresa multinacional. o\&s, v. 14, n. 43, p. 141-158, out/nov 2007.

GIL, A. C. Como elaborar projetos de pesquisa. São Paulo: Atlas, 2010.

JONES, T. A. UK hotel operators use of budgetary procedures. International Journal of Contemporary Hospitality Management, Vol. 10 (3), p. 96-100, 1998.

JONES, T. A. Changes in hotel industry budgetary practice. International Journal of Contemporary Hospitality Management, v. 20, n. 4, p. 428-444, 2008.

JOSHI, P. L.; AL-MUDHAKI, J.; BREMSER, W. G. Corporate budget planning, control and performance evaluation in Bahrain. Managerial Auditing Journal, v. 18, n. 9, p. 737-750, 2003.

LEITE, R. M.; CHEROBIM, A. P. M. S.; SILVA, H. F. N.; BUFREM, L. S. Orçamento empresarial: levantamento da produção científica no período de 1995 e 2006. Revista Contabilidade e Finanças, v. 19, n. 47, p. 56-72, 2008.

LUNKES, R. J. Contabilidade gerencial: um enfoque na tomada de decisão. Florianópolis: Visual Books, 2007. 256 p.

LUNKES, R. J.; CODESSO, M. M.; RIPOLL-FELIU, V. Práticas Orçamentárias: Um Estudo em Portos do Sul do Brasil. Contabilidad y Negocios, v. 9, p. 41-57, 2014.

LUNKES, R. J.; BELLI, A. P.; ANDRUCHECHEN, J. R.; SUAVE, R. . Práticas de Orçamento no Setor de Bens Indústriais. RACEF - Revista de Administração, Contabilidade e Economia da FUNDACE, v. 9, p. 1-17, 2014.

PAPA, V.; RIQUENA, C. A. Gestão de Concessionários de Veículos. 1 ${ }^{\text {a }}$. ed. São Paulo: Alaúde Editoral, 2011.

PAVLATOS, O.; PAGGIOS, I. Management accounting practices in the Greek hospitality industry. Managerial Auditing Journal, v. 24, n. 1, p. 81-98, 2009.

PEREIRA, V.M; LYRIO, M.V.L; LUNKES, R.S; SCHNORRENBERGER, D. Estudo das práticas orçamentárias de cooperativas agropecuárias de santa catarina. Revista em Agronegócio e Meio Ambiente, Maringa-PR, v. 8, n. 1, p. 31-56, jan/abr 2015. ISSN 2176- 
9168.

RICHARDSON, R. J. Pesquisa social: métodos e técnicas. 3. ed. São Paulo: Atlas, 1999.

SCHMIDGALL, R. S.; BORCHGREVINK, C. P.; ZAHL-BEGNUM, O. H. Operations budgeting practices of lodging firms in the United States and Scandinavia. International Journal of Hospitality Management, 15 (2), p. 189-203, 1996.

SCHMIDGALL, R.S.; DEFRANCO, A.L. Budgeting and forecasting: Current practice in the lodging industry. Cornell Hotel and Restaurant Administration Quarterly, 39 (6), p. 45 $51,1998$.

SCHMIDGALL, R.S., NINEMEIER, J. Budgeting practices in lodging and food service chains: an analysis and comparison. International Journal of Hospitality Management, 8 (1), p. 35-41, 1989.

SCHNORRENBERGER, D.; LUNKES, R. J.; DAITX, R. R. Práticas de Orçamento em Empresas Cerâmicas do Sul do Brasil. Cerâmica Industrial (Impresso), v. 19, p. 1-8, 2014.

SUAVE, R.; PINTO, H. M.; CODESSO, M. M.; LUNKES, R. J. Práticas de orçamento nos processos de planejamento e execução: um estudo em bancos comerciais públicos do Brasil. In: XX Congresso Brasileiro de Custos, 2013, Uberlândia. XX Congresso Brasileiro de Custos. Uberlândia: ABC, 2013. v. 1. p. 1-15.

SUBRAMANIAM, N.; MCMANUS, L.; MIA, L. Enhancing hotel manager's organisational commitment: An investigation of the impact of structure, need for achievement and participative budgeting. International Journal of Hospitality Management, v. 21, n. 4, p. 303-320, 2002.

STEED, E.; GU, Z. Hotel management company forecasting and budgeting practices: a survey-based analysis. International Journal of Contemporary Hospitality Management, v.21, n.6, p.676-697, 2009.

UYAR, A.; BILGIN, N. Budgeting practices in the Turkish hospitality industry: an exploratory survey in the Antalya region. International Journal of Hospitality Management, v. 30, n. 2, p. 398-408, 2011.

WELSCH, G. A. Orçamento empresarial. 4. ed. São Paulo: Atlas, 1973/1983/1989.

WINATA, L.; MIA, L. Information technology and the performance effect of managers' participation in budgeting: Evidence from the hotel industry. International Journal of Hospitality Management, v. 24, n. 1, p. 21-39, 2005.

YUEN, D. The impact of a budgetary design system: Direct and indirect models. Managerial Auditing Journal, v. 21, n. 2, p. 148-165, 2006. 